\title{
Thread Couplings Stress Analysis by Radial Basis Functions Mesh Morphing
}

\author{
Michele Calì ${ }^{1}(\bowtie)$ (10), Salvatore Massimo Oliveri ${ }^{1}$ (D), \\ and Marco Evangelos Biancolini ${ }^{2}$ \\ ${ }^{1}$ Department of Electric, Electronics and Computer Engineering, University of Catania, \\ Catania, Italy \\ michele.cali@dieei.unict.it \\ 2 Department of Enterprise Engineering "Mario Lucertini”, University of Rome "Tor Vergata”, \\ Rome, Italy
}

\begin{abstract}
Traditional analytical methods are approximate and need to be validated when it comes to predict the tensional behavior of thread coupling. Numerical finite element simulations help engineers come up with the optimum design, although the latter depends on the constraints and load conditions of the thread couplings which are often variable during the system functioning. The present work illustrates a new method based on Radial Basis Functions Mesh Morphing formulation to optimize the stress concentration in thread couplings which is subject to variable loads and constraints. In particular, thread root and fillet under-head drawings for metric ISO thread, which are the most commonly used thread connection, are optimized with Radial Basis Functions Mesh Morphing. In metric ISO threaded connection, the root shape and the fillet under the head are circular, and from shape optimization for minimum stress concentration it is well known that the circular shape becomes seldom optimal. The study is carried out to enhance the stress concentration factor with a simple geometric parameterization using two design variables. Radial Basis Functions Mesh Morphing formulation, performed with a simple geometric parameterization, has allowed to obtain a stress reduction of up to $12 \%$; some similarities are found in the optimized designs leading to the proposal of a new standard. The reductions in the stress are achieved by rather simple changes made to the cutting tool.
\end{abstract}

Keywords: Thread root $\cdot$ Fillet under-head $\cdot$ Feature-based modeling $\cdot$ Metric ISO thread · Radial basis functions

\section{Introduction}

All industrial applications commonly use threaded couplings as, for example, in transport, mining, automotive, offshore industry and in manufacturing process and more recently in dental implants. The evaluation of the stress distribution in threaded coupling is a classic topic in mechanical design. The oldest scientific papers [1-3] date from the first half of the 20th century; they focus correctly the main problems: the nonuniform load distribution on the engaged threads, the stress concentration at the root of 
the threads and under the head of the screw. As reported in [4, 5], failures in screws can be so divided: $15 \%$ at the fillet under the head, $20 \%$ at the first thread and $65 \%$ at the first engaged thread.

Analytical evaluation and numerical finite element (FE) simulations of the stress distribution on the coil are applied in different fields, such as in the design of machinery organs and in their assembly [6,7], in the biomedical field to assess the influence of thread shape on the mechanical behavior of bone implant systems [8], in the reliability field $[9,10]$.

In this work Radial Basis Functions (RBF) Mesh Morphing (MM) formulation was applied to construct, directly in the FE environment, a parametric model of a bolted couplings with axial and sheared variable loads with the aim of optimizing the stress concentration in thread root and fillet under-head. RBF were employed in literature to carry shape-based optimization [11], but their use was also explored for advanced studies, such as steady $[12,13]$ and unsteady [14] fluid structure interaction problems, evaluative shape-based optimizations $[15,16]$, etc.

The advantages of this approach are: no need to regenerate the grid; the preservation of robustness of the procedure; the capability to support different mesh typologies thanks to its meshless nature and the high parallelizable smoothing process. The morphing action integrated in a solver updating the computational domain "on the fly" during the progress of the computation can be executed in RBF Morph across three steps: 1) setup: that is the manual definition of the morphing targets, i.e. the portions of the FEA mesh that will be updated, and morphing sources, i.e. the portions of the FEA mesh controlled, and the definition of the required movements (design parameters) of the points driving the shape deformation; 2) fitting: the solution of RBF system obtained by collecting the morphing sources; 3) smoothing: by propagating the displacement prescribed on sources to the volume mesh target. Using the commercial tools ANSYS Mechanical ${ }^{\circledR}$ and the companion RBF Morph ${ }^{\mathrm{TM}}$ ACT Extension plugin, the mentioned three steps were performed as explained in the following sections.

\section{Discretized Models}

Two FE models were built for M12 and M16 bolt-nut assembly. The models employ 128116 second order tetrahedrons (TET 10) elements SOLID 187 with 254359 nodes for the bolt and 98116 TET 10 elements with 153259 nodes for the nut. In the thread root and fillet under-head contact zone a more dense mesh with patch conformity was employed using element CONTA 174 and TARGA 170. These elements allow to simulate the friction forces in the contacts. Using Remote Point formulation axial $\mathrm{F}$ and transversal T external loads (variable between $50 \mathrm{kN}$ and $80 \mathrm{kN}$ and $10 \mathrm{kN}$ and $20 \mathrm{kN}$, respectively) were applied on a dependent node (center of the spherical zone) and distributed on 36 independent nodes (within the spherical area). In this way the loads can be applied in a realistic way without stress peaks. A preload calculated with the formula:

$$
F_{\text {preload }}=0.8 \sigma_{s} \mathrm{~A}_{\mathrm{t}}
$$

was applied in the assembly systems. The preload was applied such that the stress due to it is equal to $80 \%$ of the maximum admissible stress. With this preload the assembled 
elements do not have relative movement due to the application of external loads acting in the normal direction, and do not have relative movement of slipping due to the shear forces. The discretized models are shown in Fig. 1(a) and 1(b); in Fig. 1(c) loads, preload and constrains section scheme is shown.

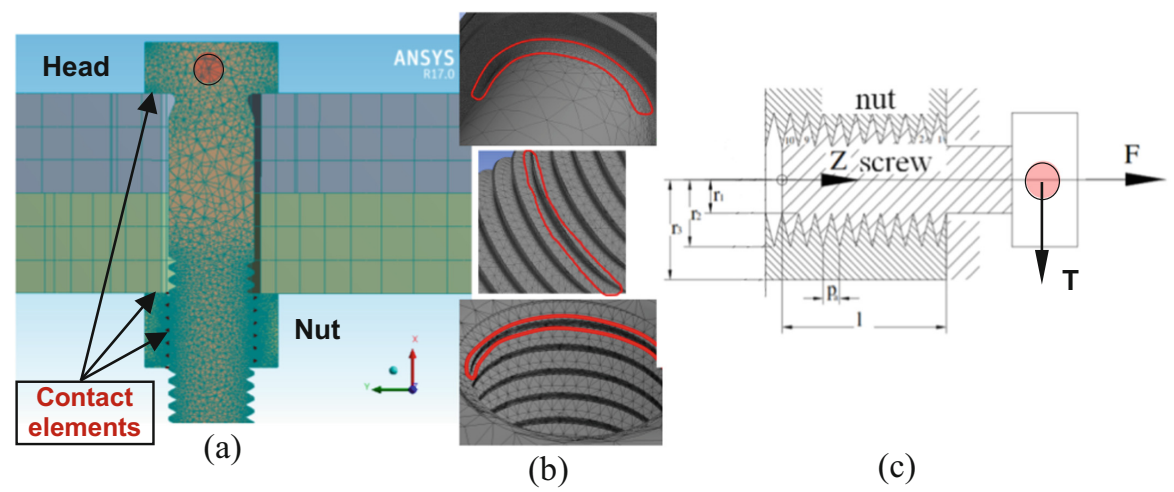

Fig. 1. (a) Bold-nut connection model; (b) optimization zones; (c) load section scheme.

\section{Parameterization}

Geometric parameterization based on RBF-MM implements shape modifiers directly on the computational domain. The new geometric configurations resulted from the displacement of a mesh region, through the use of algorithms, based on RBFs, which smoothly propagated the imposed displacement to the surrounding volume. The fitting size in the thread root and fillet under-head, according to current standard (UNI EN ISO 4016), are calculated as follows:

$$
R_{\text {min }}=\frac{\mathrm{H}}{8}=0.1082 P
$$

being $\mathrm{H}$ the height of the non-fillet profile (Fig. 2a): $\mathrm{H}=\sin 60^{\circ} P=0.866 P$

Triple multicenter circular fillets were used as guide curves for profile at thread root and for profile under-head. Five parameters $\left(\mathrm{R}_{1}, \mathrm{R}_{2}, \mathrm{R}_{0}{ }_{0}\right.$ and $\left.\mathrm{R}^{\prime}{ }_{1}, \mathrm{R}_{2}{ }_{2}\right)$ defining the curves radii were varied at given boundary conditions and mutual relationships to generate a different profile allowed by standards. The RBF interpolation functions drove the mesh morphing of the discretized domain of computational models by applying predefined displacements in the set of 2600 nodes (source points) near the fillet zones without changing their number of the topology of the mesh. During the mesh morphing the nodes on the profile moved according to the defined constrain varying the $R_{1}$ and $R_{2}$ fillet radius parameters (Fig. 3).

Then the RBF action was extended in the entire volume, smoothing the grid up to the prescribed surrounding limits. The obtained RBF solution was sent to the ANSYS Mechanical solver and an iterative calculation strategy was chosen. 


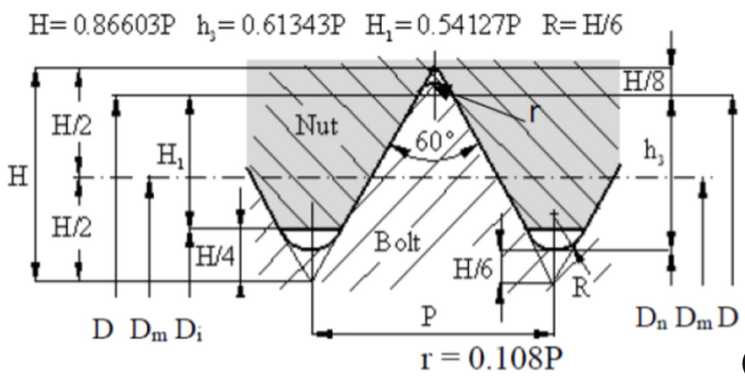

(a)
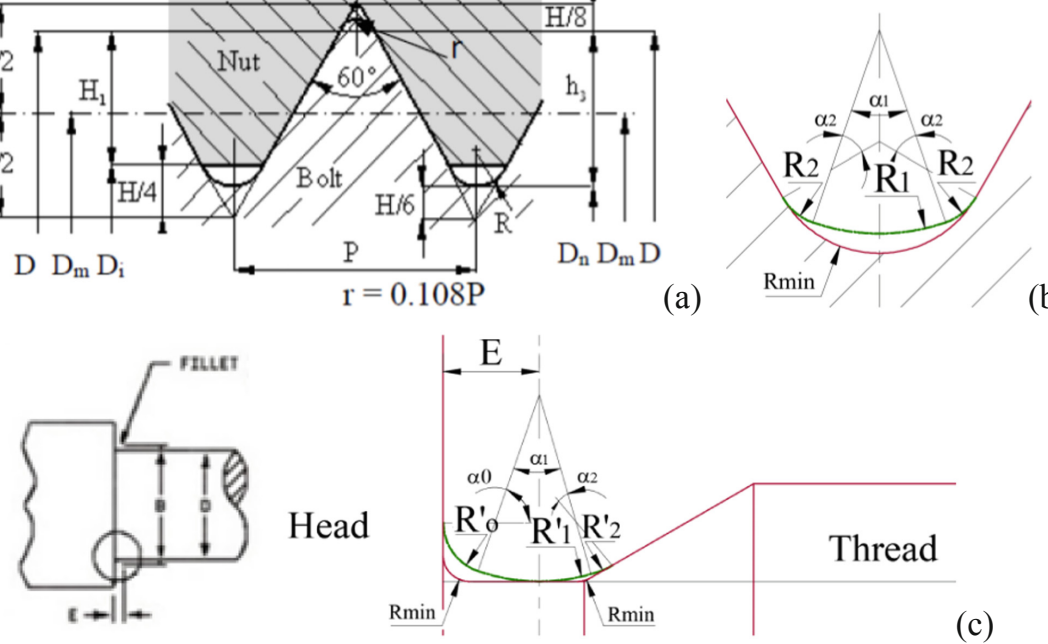

(c)

Fig. 2. (a) Geometrical features of metric ISO thread coupling; (b) particular of fillet at thread root; (c) particular of fillet under-head.

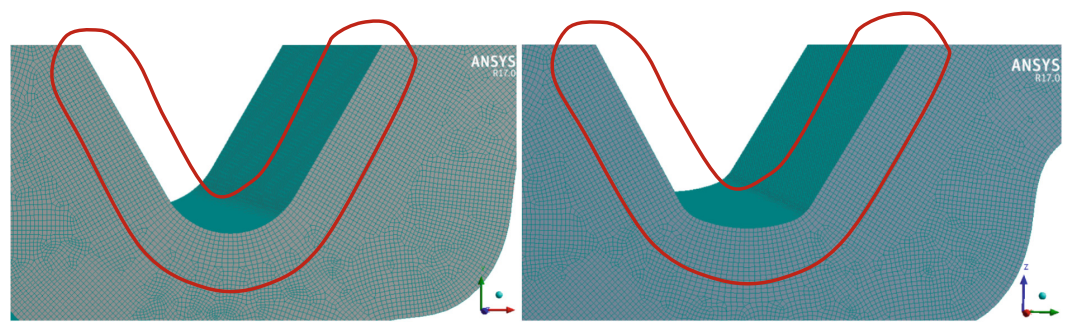

Fig. 3. Source points in the thread root.

\section{Results and Discussion}

According to the RBF-MM formulation and parameterization described above four standard metric ISO thread couplings: M12 × 1.25; M12 × 1.75; M16 × 1.5; M16 × 2 were morphed under axial $\mathrm{F}$ and transversal $\mathrm{T}$ variable loads $(50 \mathrm{kN} \div 80 \mathrm{kN}$ and $10 \mathrm{kN} \div$ $20 \mathrm{kN}$, respectively). The set of parameters and their variability shown in Table 1 were chosen for thread root analysis. The set of parameters and their variability shown in Table 2 were chosen for fillet under-head root analysis. The theoretical approach used to calculate the maximum von Mises stress is the method of Heywood [4] and Pilkey [5]. The maximum von Mises stress value was calculated evaluating the four components of the stress state: axial, radial, azimuthal, and shear stresses using respectively the following formulas:

$$
\sigma_{a x}=\frac{F}{A} K_{t}(A x) ; \sigma_{R a d}=\left(\frac{F\left(\frac{H}{2}+\frac{p}{4}\right)}{W}+\frac{F}{A}\right) K_{t}(\operatorname{Rad}) ;
$$




$$
\sigma_{a z}=v\left(\sigma_{a x}+\sigma_{R a d}\right) ; \tau=\frac{F^{2}}{A} \frac{k(A x)}{1+q\left(\frac{\sigma_{a x}}{\sigma_{\text {Rad }}}\right)}
$$

where $\mathrm{K}_{\mathrm{t}}(\mathrm{Ax}), \mathrm{K}_{\mathrm{t}}(\mathrm{Rad})$ and $\mathrm{q}$ values are taken from the Pilkey's diagrams [5]. In this way it was possible to calculate the von Mises stress using the formula:

$$
\sigma_{V M}=\sqrt{\sigma_{a x}^{2}+\sigma_{R a d}^{2}+\sigma_{a z}^{2}-\sigma_{a x} \sigma_{R a d}-\sigma_{a x} \sigma_{a z}-\sigma_{R a d} \sigma_{a z}+3 \tau^{2}}
$$

In correspondence with the maximum values of $\mathrm{F}$ and $\mathrm{T}(\mathrm{F}=80 \mathrm{kN}$ and $\mathrm{T}=20 \mathrm{kN})$ were calculated maximum von Mises stress in thread root and in fillet.

Table 1. FEM and theoretical values of maximum von Mises stresses in thread root.

\begin{tabular}{l|l|l|l|l}
\hline Case studies & $\mathrm{R}_{1}[\mathrm{~mm}]$ & $\mathrm{R}_{2}[\mathrm{~mm}]$ & FEM values & Theoretical values \\
\hline $\mathrm{M} 12 \times 1.25$ & $0.135 \div 0.406$ & $0.02 \div 0.06$ & 558.60 & 565.36 \\
\hline $\mathrm{M} 12 \times 1.75$ & $0.189 \div 0.568$ & $0.03 \div 0.09$ & 548.26 & 544.55 \\
\hline $\mathrm{M} 16 \times 1.5$ & $0.162 \div 0.487$ & $0.025 \div 0.075$ & 520.18 & 514.59 \\
\hline $\mathrm{M} 16 \times 2$ & $0.216 \div 0.649$ & $0.033 \div 0.1$ & 482.5 & 489.08 \\
\hline
\end{tabular}

Table 2. FEM and theoretical values of maximum von Mises stresses in fillet under-head.

\begin{tabular}{l|l|l|l|l|l}
\hline Case studies & R'0 $\left._{0}^{\prime} \mathrm{mm}\right]$ & R'$_{1}^{\prime}[\mathrm{mm}]$ & $\mathrm{R}_{2}^{\prime}[\mathrm{mm}]$ & FEM values & Theoretical values \\
\hline M12 $\times 1.25$ & $0.135 \div 0.406$ & $1.5 \div 4$ & $0.4 \div 2$ & 448.64 & 454.23 \\
\hline M12 $\times 1.75$ & $0.189 \div 0.568$ & $2 \div 6$ & $0.6 \div 3$ & 419.82 & 424.48 \\
\hline M16 $\times 1.5$ & $0.162 \div 0.487$ & $1.7 \div 4.5$ & $0.5 \div 2.2$ & 468.99 & 473.07 \\
\hline M16 $\times 2$ & $0.216 \div 0.649$ & $2.5 \div 7$ & $0.8 \div 3.5$ & 393.67 & 389.16 \\
\hline
\end{tabular}
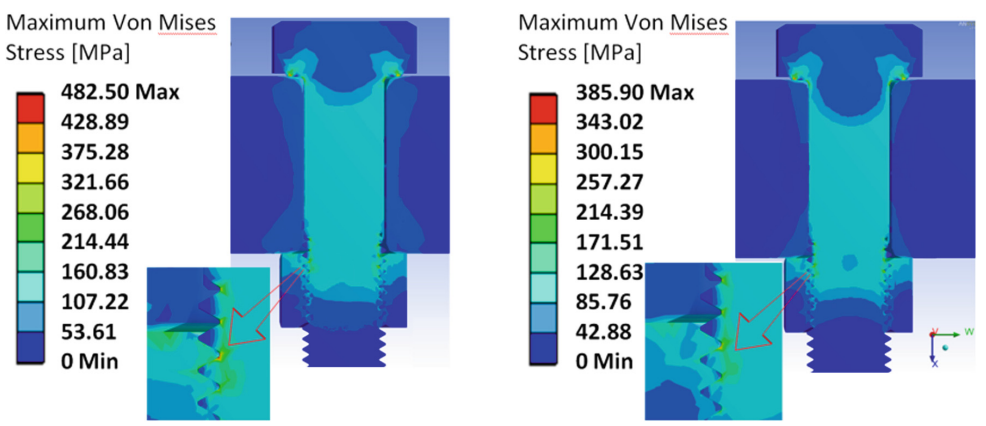

Fig. 4. Maximum equivalent von Mises stress: (a) standard geometry; (b) modified geometry.

The increase in the fillet radii led to a better load distribution and a decrease of maximum von Mises stress of about $15 \%$. The greatest improvement (20\%) occurred in 
the M16 $\times 2$ thread couplings using the values of $\mathrm{R}_{1}=0.649 ; \mathrm{R}_{2}=0.033 ; \mathrm{R}_{0}=0.216$; $\mathrm{R}^{\prime}{ }_{1}=7$ and $\mathrm{R}_{2}{ }_{2}=0.8$; the maximum von Mises stress was reduced to $385.90 \mathrm{MPa}$ corresponding to the $60 \%$ of limit stress $\left(\sigma_{\mathrm{r}}=800 \mathrm{~N} / \mathrm{mm}^{2} ; \sigma_{\mathrm{s}}=640 \mathrm{~N} / \mathrm{mm}^{2} \mathrm{E}=\right.$ $200 \mathrm{kN} / \mathrm{mm}^{2}$ ) for 8.8 class of bolt. Figure 4 shows the values of the maximum von Mises stress and their reduction after optimization for this thread.

\section{Conclusions}

A Radial Basis Functions Mesh Morphing formulation was adopted in order to create parametric models of bolted couplings with axial and shear variable loads directly in the FE environment, aimed at optimizing the maximum stress in thread couplings. The optimal drawing of thread root and fillet under-head was found. Four case studies demonstrated its effectiveness optimizing the maximum equivalent von Mises stress. It was possible to reduce the peak load and the maximum stress, enlarging the internal diameter of the thread root with triple multicenter circular fillets so improving the strength and fatigue life by means of a simple and not expensive modification. The comparison with theoretical data showed a really negligible maximum error on load distribution and an improvement of the threaded coupling. Finally, the calculations performed on the two diameters seemed to demonstrate that the improving effect was greater for the largest diameter.

Acknowledgments. The research work herein reported was funded by Università degli Studi di Catania within the CRUI-CARE Agreement (research path PIA.CE.RI. 2020-2022 Linea 3 STARTING GRANT 2020) and by "Smart main. of ind. Plants and civ. Struct. by 4.0 monit. Tech. And pron. Appr.-MAC4PRO”.

\section{References}

1. Stromayer, C.E.: Stress distribution in bolts and nuts. Trans. Last. Nav. Arch. 60, 112-121 (1918)

2. Den Hartog J.P. The mechanics of plate rotors for turbo-generators, Trans of ASME, 51(1), 1-10 (1929)

3. Goodier, J.N.: The distribution of load on threads of screws. J. Appl. Mech. 93-100 (1940)

4. Heywood, R.B.: Designing by Photoelasticity. Chapman and Hall, London (1952)

5. Pilkey, W.D., Pilkey, D.F.: Peterson's Stress Concentration Factors. J. Wiley and S, N.Y. (2008)

6. Pedersen, N.L.: Optimization of bolt thread stress concentrations. Arch. Appl. Mech. 83(1), 1-14 (2013)

7. Ball, R.S.: A Treatise on the Theory of Screws. Cambridge University Press, Cambridge (1998)

8. Zanetti, E.M., Ciaramella, S., Calì, M., Pascoletti, G., Martorelli, M., Asero, R., Watts, D.C.: Modal analysis for implant stability assessment: sensitivity of this methodology for different implant designs. Dent. Mater. 34(8), 1235-1245 (2018)

9. Birger, I.A.: Load distribution on thread in threaded joints. Bull. Mach. Eng. I (1944) 
10. Biancolini, M.E.: Fast radial basis functions for engineering applications. Springer (2017)

11. Biancolini, M.E., Costa, E., et al.: Glider fuselage-wing junction optimization using CFD and RBF mesh morphing. Aircraft Eng. Aerosp. Technol. 88, 740-752 (2016)

12. Pascoletti, G., Calì, M., Bignardi, C., Conti, P., Zanetti, E.M.: Mandible morphing through principal components analysis. Lecture Notes in Mech. Engineering, pp. 15-23. Springer (2020)

13. Calì, M., Oliveri, S.M.: Application of an effective SIMP method with filtering for topology optimization of motorcycle tubular frame. Int. Rev. Mech. Eng. 11(11), 836-844 (2017)

14. Di Domenico, N., Groth, C., Wade, A., Berg, T., Biancolini, M.E.: Fluid structure interaction analysis: Vortex shedding induced vibrations. Procedia Struct. Integrity 422-432 (2018)

15. Calì, M., Oliveri, S.M., Cella, U., Martorelli, M., Gloria, A., Speranza, D.: Mechanical characterization and modeling of downwind sailcloth in fluid-structure interaction analysis. Ocean Eng. 165, 488-504 (2018)

16. Calì, M., Speranza, D., Cella, U., Biancolini, M.E.: Flying shape sails analysis by radial basis functions mesh morphing. Lecture Notes in Mechanical Engineering, pp. 24-36. Springer (2020)

Open Access This chapter is licensed under the terms of the Creative Commons Attribution 4.0 International License (http://creativecommons.org/licenses/by/4.0/), which permits use, sharing, adaptation, distribution and reproduction in any medium or format, as long as you give appropriate credit to the original author(s) and the source, provide a link to the Creative Commons license and indicate if changes were made.

The images or other third party material in this chapter are included in the chapter's Creative Commons license, unless indicated otherwise in a credit line to the material. If material is not included in the chapter's Creative Commons license and your intended use is not permitted by statutory regulation or exceeds the permitted use, you will need to obtain permission directly from the copyright holder. 